\title{
ChemComm
}

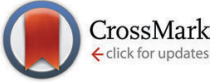

Cite this: Chem. Commun., 2016, 52,12179

Received 18th August 2016

Accepted 15th September 2016

DOI: $10.1039 / c 6 c c 06800 f$

www.rsc.org/chemcomm

\section{Magnesium plasmonics for UV applications and chiral sensing $\dagger+$}

\author{
Hyeon-Ho Jeong, ${ }^{\text {ab }}$ Andrew G. Mark ${ }^{a}$ and Peer Fischer*ac
}

\begin{abstract}
We demonstrate that chiral magnesium nanoparticles show remarkable plasmonic extinction- and chiroptical-effects in the ultraviolet region. The $\mathrm{Mg}$ nanohelices possess an enhanced local surface plasmon resonance (LSPR) sensitivity due to the strong dispersion of most substances in the UV region.
\end{abstract}

Nanoparticles whose size is smaller than the wavelength of light can give rise to light-induced collective electron oscillations, known as local surface plasmon resonance (LSPR). ${ }^{1,2}$ The resonance wavelength in LSPR is sensitive to the environment, which is the basis for a number of sensing applications. ${ }^{2}$ Improvements in sensitivity have been achieved by engineering the particles' size, shape, and material composition, ${ }^{3,4}$ and the mode of detection. ${ }^{5,6}$ Studies to date have primarily focussed on increasing the shift of the resonance for a given change in the refractive index. However, in many applications the quantity of ultimate interest is not the medium's refractive index, but the concentration of some chemical species. ${ }^{7,8}$ One particularly promising route to improve the concentration sensitivity is to exploit the natural refractive index dispersion of the molecule (analyte) of interest. Most molecules show notably higher and more dispersive refractive indices at short wavelengths, because of electronic resonances (transitions) in the UV region. Changes in the concentration of the analyte therefore yield larger changes in the refractive index at shorter wavelengths, and therefore larger shifts in the plasmon resonance. However, this also requires a plasmonic probe that shows a UV resonance.

Here, we introduce what we believe are the first magnesium $(\mathrm{Mg})$ nanoparticles for UV plasmonics and LSPR sensing. The $\mathrm{Mg}$ nanoparticles are grown with a helical shape. Their exceptionally

\footnotetext{
${ }^{a}$ Max Planck Institute for Intelligent Systems, Heisenbergstr. 3, 70569 Stuttgart, Germany

${ }^{b}$ Institute of Materials, École Polytechnique Fédérale de Lausanne (EPFL), CH-1015 Lausanne, Switzerland

${ }^{c}$ Institute for Physical Chemistry, University of Stuttgart, Pfaffenwaldring 55, 70569 Stuttgart, Germany. E-mail: fischer@is.mpg.de

$\dagger$ Electronic supplementary information (ESI) available. See DOI: 10.1039/c6cc06800f \$ Part of the issue "chirality at the Nanoscale".
}

strong chiroptical response in the near UV region (e.g. circular dichroism, $\mathrm{CD}=\sim 13^{\circ}$ at $\lambda=\sim 437 \mathrm{~nm}$ in solution, see the ESI, $\dagger$ Fig. S2) gives rise to a concomitant enhancement of the LSPR sensitivity.

In an earlier study, ${ }^{5}$ we developed a chiral plasmonic sensing approach that is based on a chiral nanoprobe (i.e. it measures the differential extinction between left-handed (LH) and righthanded (RH) circularly polarised light using CD spectroscopy, i.e. $\left.\mathrm{CD} \propto E_{\mathrm{L}}-E_{\mathrm{R}}\right)$. The change in response to the change in the refractive index of the medium surrounding the nanoparticles is tracked, as schematically shown in Fig. 1. CD-based sensing with chiral nanoparticles offers two distinct advantages over traditional polarisation independent measurements: firstly, the CD signal of a chiral absorber naturally comprises multiple spectral features compared to an extinction peak, and each feature can be tracked. This provides high sensing flexibility and fidelity. In particular, the zero crossings $\left(\mathrm{CD}=0^{\circ}\right)$ where the $\mathrm{LH}$ and $\mathrm{RH}$ extinctions are equal are ideal for tracking, because they are intrinsically narrow and possess high figures of merit. ${ }^{5}$ Secondly, the chiral particles provide a strong optical contrast, even in the presence of optically dense fluids, as any achiral background signals are cancelled. Hence, chiral nanoparticles can even be "seen" in strongly absorbing physiological fluids (e.g. blood). ${ }^{9}$

The chiral LSPR sensor operates by tracking the shift in the zero crossing of the CD signal in response to changes in $n$, the refractive index of the local medium, which surrounds the particles. The resonance condition is ${ }^{5}$

$$
\varepsilon_{\mathrm{r}}\left(\lambda^{*}\right)=-\bar{\chi} n^{2},
$$

where $\lambda^{*}$ is the resonance wavelength at zero crossing, $\varepsilon_{\mathrm{r}}$ is the real part of the dielectric function of the plasmonic nanoparticle, and $\bar{\chi}$ is a shape-dependent factor. For sensing tasks undertaken in the visible to NIR regime typical of conventional plasmonics, eqn (1) is reasonable (ESI, $\dagger$ Fig. S1). ${ }^{8,10}$ However, for measurements in the UV region, the dispersion of the medium (solvent and analyte) $n(\lambda)$ needs to be considered, such that

$$
\varepsilon_{\mathrm{r}}\left(\lambda^{*}\right)=-\bar{\chi}\left[n\left(\lambda^{*}\right)\right]^{2} .
$$



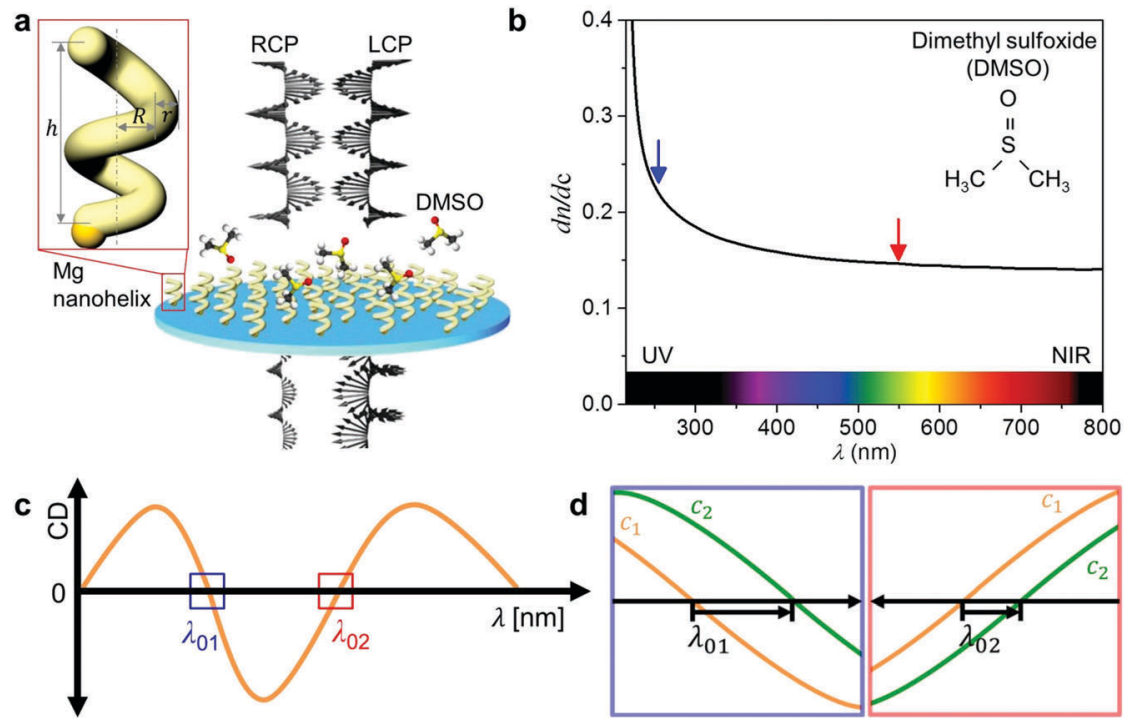

Fig. 1 Sensing using UV chiral plasmonics: (a) schematic that illustrates the interaction of left-handed (LH) nanohelices with left- and right-handed circularly polarised light (LCP, RCP) in the presence of dimethyl sulfoxide (DMSO). The inset shows the schematic view of a 2 -turn left-handed Mg nanohelix showing dimensions of the seed particle and the nanostructure that grows upon it ( $h$ : height, $R$ : major radius, $r$ : minor radius). (b) Change in the refractive index of the DMSO solution in response to a change in its concentration ( $\mathrm{d} n \cdot \mathrm{dc} c^{-1}$ ) as a function of $\lambda_{1}^{12,13}$ which is larger in the UV (blue arrow) than in the visible (red arrow) region. (c) A CD spectrum of LH nanohelices as a function of $\lambda$. Two boxes indicate zero crossing points, $\lambda_{01}$ (blue: in UV) and $\lambda_{02}$ (red: in visible). (d) Each panel indicates the resonance shifts at $\lambda_{01}$ (left panel) and $\lambda_{02}$ (right panel) where the concentrations of the molecules are varied between $c_{1}$ and $c_{2}\left(c_{1}<c_{2}\right)$.

In LSPR sensing it is customary to define a refractive index sensitivity, which describes the change in the resonance position (wavelength) as a function of refractive index ${ }^{5}$

$$
S_{n}=\frac{\mathrm{d} \lambda^{*}}{\mathrm{~d} n}=\frac{-2 \bar{\chi} n\left(\lambda^{*}\right)}{\frac{\mathrm{d} \varepsilon_{\mathrm{r}}\left(\lambda^{*}\right)}{\mathrm{d} \lambda}} .
$$

However, another important factor in assessing sensitivity is to determine by how much the tracking wavelength $\lambda^{*}$ changes in response to the volume concentration $c$ of the species of interest

$$
\frac{\mathrm{d} \lambda^{*}}{\mathrm{~d} c}=\frac{\mathrm{d} \lambda^{*}}{\mathrm{~d} n} \frac{\mathrm{d} n}{\mathrm{~d} c}=S_{n} \frac{\mathrm{d} n}{\mathrm{~d} c}
$$

The refractive index of a mixture of a solute and a solvent with indices $n_{1}$ and $n_{2}$ can be approximated, using the rule of Arago and Biot, as ${ }^{11}$

$$
n=n_{2} c+n_{1} \cdot(1-c)
$$

SO

$$
\frac{\mathrm{d} n}{\mathrm{~d} c}=n_{2}-n_{1}
$$

Consider dimethyl sulfoxide (DMSO) in water (Fig. 1b). ${ }^{12,13}$ Within the visible region the refractive index of DMSO is everywhere higher than that of water, so the sign of eqn (6) is always positive. But $\mathrm{d} n \cdot \mathrm{d} c^{-1}$ at $600 \mathrm{~nm}$ is only $0.144 \mathrm{RIU}$, whereas at $250 \mathrm{~nm}$ it is 0.229 RIU, almost $60 \%$ higher. Thus, enhanced sensitivity to changes in solute concentration (eqn (4)) can be achieved at shorter wavelengths, and in particular through measurements in the UV region.
Aluminium (Al) nanoparticles have thus far been favoured for UV plasmonic applications, ${ }^{14,15}$ even though theory predicts that $\mathrm{Mg}$ provides substantially higher far-field absorption efficiency and higher near-field electric field enhancements. ${ }^{16}$ Furthermore, $\mathrm{Mg}$ is abundant, biocompatible, and lightweight (twothirds lighter than Al). ${ }^{17}$ However, to the best of our knowledge, there is no report of $\mathrm{Mg}$ nanoparticles for UV plasmonics. ${ }^{18}$ Here we show how we can grow large numbers of chiral $\mathrm{Mg}$ nanoparticles using a previously reported fabrication method that we term nano glancing angle deposition (nanoGLAD), which allows precise control of size, shape, and material composition of the nanoparticles at the wafer-level. ${ }^{19-21}$ Briefly, an array of gold nanoseeds is first patterned on a $1 \mathrm{~cm}^{2}$ quartz glass using block-copolymer micelle nanolithography (BCML). ${ }^{22}$ Then, in a second step, $\mathrm{Mg}$ is evaporated onto the Au nanoseeds at an angle $\alpha=87^{\circ}$ and $T=\sim 100 \mathrm{~K}$ in a vacuum chamber with a base pressure $P=1 \times 10^{-6}$ mbar. $^{23-28}$ The substrate is continuously rotated during deposition, which, because of the high angle, leads to the growth of a nanohelix on each $\mathrm{Au}$ nanoseed by a selfshadowing effect. ${ }^{19,29,30}$ Due to the fast diffusion of the $\mathrm{Mg}$ adatoms, we were not able to shape nanohelices with pure $\mathrm{Mg}$ (see the ESI, $\dagger$ Fig. S2), so we co-deposited $8 \%$ of titanium (Ti). ${ }^{31}$ Doping $\mathrm{Ti}$ into the $\mathrm{Mg}$ matrix does not significantly change the optical properties, but it improves the structural quality of the nanostructures. ${ }^{32}$ Moreover, as the nanoparticle becomes more Ti rich, the LSPR sensitivity is enhanced since Ti flattens the real part of the dielectric function of the composite. ${ }^{5}$ The increase in damping also somewhat broadens the lines, but this effect does not diminish the overall improvement. ${ }^{33}$ Finally, the nanohelices are coated with a thin layer of $\mathrm{HfO}_{2}$ by atomic layer deposition (ALD, Savannah 100, Cambridge NanoTech). ${ }^{34}$ The $4 \mathrm{~nm}$ thin 
oxide layer improves the chemical resistance of the particles. ${ }^{14}$ Fig. 2a shows scanning electron microscopy (SEM) images of arrays of two different chiralities of $8 \%$ Ti-doped $\mathrm{Mg}$ nanohelices (here samples are prepared on Si wafers for imaging). Helices with opposite chiralities are grown on different patterned glass substrates (as well as wafers) and during different depositions, but display very similar structural dimensions. The height, major radius, and minor radius of the helices are $142 \mathrm{~nm}, 26 \mathrm{~nm}$, and $18 \mathrm{~nm}$ respectively. Fig. 2b shows the extinction (upper panel) and $\mathrm{CD}$ (lower panel) spectra of $\mathrm{Mg} \mathrm{LH}$ and $\mathrm{RH}$ nanohelices obtained using a Jasco J-810 CD spectrometer. The helices exhibit chiroptical responses that saturate the spectrometer even in its largest amplitude detection range, so the responses were measured with an external lock-in-amplifier (UHFL, Zurich Instruments) referenced to the photoelastic modulator (PEM) and synchronized with the monochromator. In accordance with theoretical predictions, ${ }^{16}$ we have observed an extraordinarily large shortwavelength extinction $(\sim 1$ OD at $\lambda=\sim 385 \mathrm{~nm})$ and optical activity (CD $=\sim 8^{\circ}$ at $\lambda=\sim 380 \mathrm{~nm}$ ) (see also the ESI, $\dagger$ Fig. S3). The spectral features of both chiralities are well matched across the entire spectrum ( $\max , \mathrm{min}$, and two zero crossings).

UV-enhanced LSPR refractive index sensing is demonstrated with a DMSO-water system. In neat DMSO, CD spectra of the LH
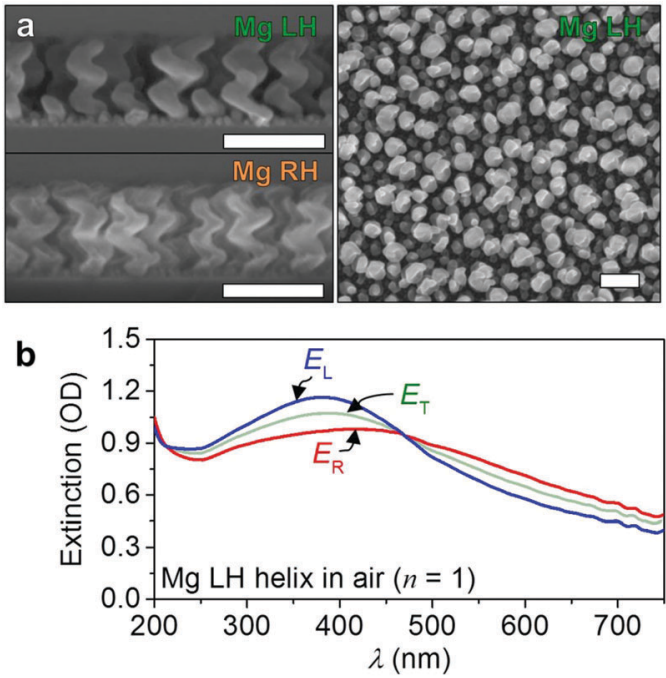

C

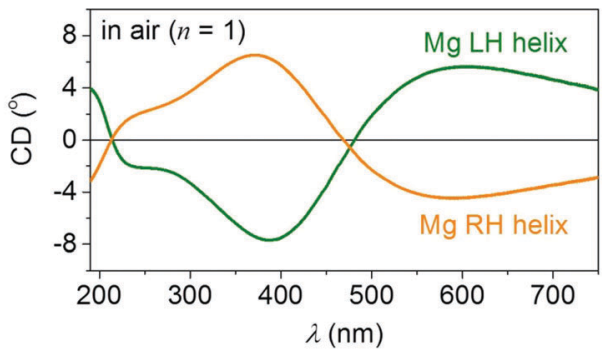

Fig. $2 \mathrm{Mg}$ nanohelices. (a) SEM images of Mg LH (left-top panel) and RH (left-bottom panel) nanohelices. The right panel shows the top view of the array of the $\mathrm{Mg} \mathrm{LH}$ nanohelices (scale bar: $200 \mathrm{~nm}$ ). (b) Extinction spectra of the $\mathrm{Mg} \mathrm{LH}$ nanohelices in air in response to the unpolarised light $\left(E_{\mathrm{T}}\right.$, green) and the circularly polarised light (LCP: $E_{\mathrm{L}}$, blue and RCP: $E_{\mathrm{R}}$, red). (c) CD spectra of the $178 \mathrm{~nm}$ thick film containing $\mathrm{LH}$ (green) and $\mathrm{RH}$ (orange) $\mathrm{Mg}$ nanohelices in air.
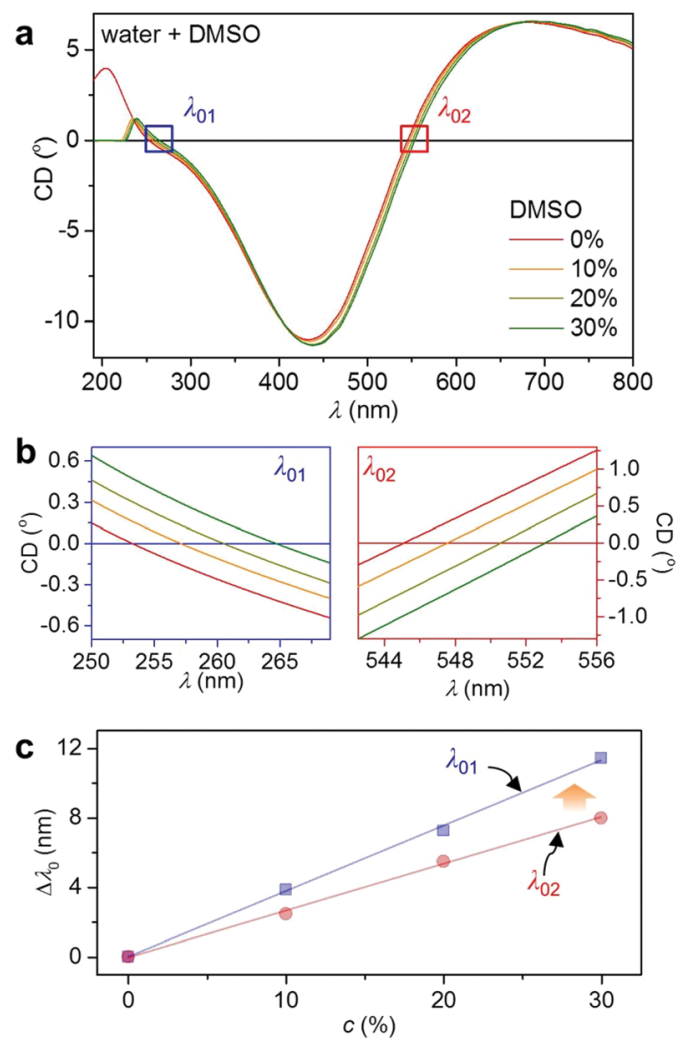

Fig. 3 Molecular LSPR sensing. (a) CD spectra of Mg LH nanohelices in media with four different refractive indices (red: 0\%, orange: $10 \%$, yellow: $20 \%$, and green: $30 \%$ DMSO-water mixtures) over the full spectral range and (b) detailed plots of the resonance shifts at $\lambda_{01}$ (left panel) and $\lambda_{02}$ (right panel). (c) Wavelength shift, relative to water, of the two spectral features as a function of the DMSO-water concentration.

$\mathrm{Mg}$ nanohelices (Fig. 3) are shifted relative to the $\mathrm{Mg}$ spectra in air (Fig. 2b), because of the relative increase of the refractive index (see Fig. 1b and the ESI, $\dagger$ Fig. S1). Fig. 3 shows CD spectra of the $\mathrm{Mg} \mathrm{LH}$ nanohelices in DMSO-water mixtures varying from 0 to $30 \%$. DMSO shows strong electronic absorption below $230 \mathrm{~nm}$. We track the CD resonances at the two zero crossings $\lambda_{01}$ and $\lambda_{02}$ (see the ESI, $\dagger$ Fig. S4 for the extinction spectra). The crossings' wavelengths shift in response to increasing DMSO concentration, as shown in Fig. 3c. Both depend linearly on the concentration of DMSO, but crucially, the shift of $\Delta \lambda_{01}$ in the UV region is larger than the shift at the longer wavelength $\Delta \lambda_{02}$. This is in agreement with the 1.6 times larger $\mathrm{d} n \cdot \mathrm{d} c^{-1}$ in the UV compared to the visible region, which in turn induces a larger plasmonic shift (see Fig. 1b). These results clearly show that UV plasmonics is promising for high sensitivity LSPR due to the dispersion of the medium.

In conclusion, this communication reports what we believe are the first $\mathrm{Mg}$ nanoparticles that have been used for plasmonics in water. $\mathrm{Mg}$ nanohelices were fabricated using nanoGLAD and their chiroptical response is exceptionally strong in the UV region. This feature is crucial for the detection of molecules as in the UV region the dispersion of the medium surrounding the plasmonic particle gives rise to larger refractive index changes. UV sensing may thus enable single molecule detection with a 
plasmonic nanosensor. ${ }^{35}$ It should be possible to increase the LSPR sensitivity further through engineering the dielectric function of $\mathrm{Mg}$ nanoparticles. ${ }^{5}$

Apart from UV sensing, Mg nanoparticles are promising for resonance coupling effects for chiral sensing ${ }^{36-39}$ and plasmon resonance energy transfer (PRET) applications, ${ }^{40-42}$ since the plasmonic resonance of $\mathrm{Mg}$ in the $\mathrm{UV}$ approaches molecular resonances.

We are grateful to the Nanostructuring Laboratory, Max Planck Institute for Solid State Research, for ALD access. We thank M. Alarcon-Correa, T.-C. Lee, and J. G. Gibbs for helpful discussions and C. Miksch for assistance with the sample preparation. This work was supported by the Max Planck-EPFL Centre for Molecular Nanoscience and Technology and the European Research Council under ERC Grant agreement 278213.

\section{Notes and references}

1 R. H. Ritchie, Phys. Rev., 1957, 106, 874-881.

2 K. A. Willets and R. P. Van Duyne, Annu. Rev. Phys. Chem., 2007, 58, 267-297.

3 H. Chen, X. Kou, Z. Yang, W. Ni and J. Wang, Langmuir, 2008, 24, 5233-5237.

4 D. E. Charles, D. Aherne, M. Gara, D. M. Ledwith, Y. K. Gun'ko, J. M. Kelly, W. J. Blau and M. E. Brennan-Fournet, ACS Nano, 2010, 4, 55-64.

5 H.-H. Jeong, A. G. Mark, M. Alarcon-Correa, I. Kim, P. Oswald, T.-C. Lee and P. Fischer, Nat. Commun., 2016, 7, 11331.

6 N. Maccaferri, K. E. Gregorczyk, T. V. A. G. de Oliveira, M. Kataja, S. van Dijken, Z. Pirzadeh, A. Dmitriev, J. Åkerman, M. Knez and P. Vavassori, Nat. Commun., 2015, 6, 6150.

7 J. N. Anker, W. P. Hall, O. Lyandres, N. C. Shah, J. Zhao and R. P. Van Duyne, Nat. Mater., 2008, 7, 442-453.

8 K. M. Mayer and J. H. Hafner, Chem. Rev., 2011, 111, 3828-3857.

9 H.-H. Jeong, A. G. Mark, T.-C. Lee, M. Alarcón-Correa, S. Eslami, T. Qiu, J. G. Gibbs and P. Fischer, Nano Lett., 2016, 16, 4887-4894.

10 M. M. Miller and A. A. Lazarides, J. Phys. Chem. B, 2005, 109, 21556-21565.

11 W. Heller, J. Phys. Chem., 1965, 69, 1123-1129.

12 I. Z. Kozma, P. Krok and E. Riedle, J. Opt. Soc. Am. B, 2005, 22, 1479-1485.

13 G. M. Hale and M. R. Querry, Appl. Opt., 1973, 12, 555-563.

14 M. W. Knight, N. S. King, L. Liu, H. O. Everitt, P. Nordlander and N. J. Halas, ACS Nano, 2014, 8, 834-840.

15 G. Maidecchi, G. Gonella, R. Proietti Zaccaria, R. Moroni, L. Anghinolfi, A. Giglia, S. Nannarone, L. Mattera, H.-L. Dai, M. Canepa and F. Bisio, ACS Nano, 2013, 7, 5834-5841.
16 J. M. Sanz, D. Ortiz, R. Alcaraz de la Osa, J. M. Saiz, F. González, A. S. Brown, M. Losurdo, H. O. Everitt and F. Moreno, J. Phys. Chem. C, 2013, 117, 19606-19615.

17 L.-Y. Chen, J.-Q. Xu, H. Choi, M. Pozuelo, X. Ma, S. Bhowmick, J.-M. Yang, S. Mathaudhu and X.-C. Li, Nature, 2015, 528, 539-543.

18 F. Sterl, N. Strohfeldt, R. Walter, R. Griessen, A. Tittl and H. Giessen, Nano Lett., 2015, 15, 7949-7955.

19 A. G. Mark, J. G. Gibbs, T.-C. Lee and P. Fischer, Nat. Mater., 2013, 12, 802-807.

20 J. G. Gibbs, A. G. Mark, T.-C. Lee, S. Eslami, D. Schamel and P. Fischer, Nanoscale, 2014, 6, 9457-9466.

21 J. G. Gibbs, A. G. Mark, S. Eslami and P. Fischer, Appl. Phys. Lett., 2013, 103, 213101.

22 R. Glass, M. Möller and J. P. Spatz, Nanotechnology, 2003, 14, 1153.

23 K. Robbie, M. J. Brett and A. Lakhtakia, Nature, 1996, 384, 616.

24 G. Nair, H. J. Singh and A. Ghosh, J. Mater. Chem. C, 2015, 3, 6831-6835.

25 H.-H. Jeong, A. G. Mark, T.-C. Lee, K. Son, W. Chen, M. AlarcónCorrea, I. Kim, G. Schütz and P. Fischer, Adv. Sci., 2015, 2, 1500016.

26 S. H. Lee, D. P. Singh, J. H. Sung, M.-H. Jo, K. C. Kwon, S. Y. Kim, H. W. Jang and J. K. Kim, Sci. Rep., 2016, 6, 19580.

27 J. Deng, J. Fu, J. Ng and Z. Huang, Nanoscale, 2016, 8, 4504-4510.

28 S. Xie, J. Yang, X. Xiao, Y. Hou, J. Du, L. Pang, X. Li and F. Gao, Nanoscale Res. Lett., 2015, 10, 1-9.

29 H.-H. Jeong, A. G. Mark, J. G. Gibbs, T. Reindl, U. Waizmann, J. Weis and P. Fischer, Nanotechnology, 2014, 25, 235302.

30 J. H. Singh, G. Nair, A. Ghosh and A. Ghosh, Nanoscale, 2013, 5, 7224-7228.

31 Y. He, Y. Zhao and J. Wu, Appl. Phys. Lett., 2008, 92, 063107.

32 G. K. Larsen, Y. He, J. Wang and Y. Zhao, Adv. Opt. Mater., 2014, 2, 245-249.

33 T. G. Habteyes, S. Dhuey, E. Wood, D. Gargas, S. Cabrini, P. J. Schuck, A. P. Alivisatos and S. R. Leone, ACS Nano, 2012, 6, 5702-5709.

34 D. Walker, B. T. Käsdorf, H.-H. Jeong, O. Lieleg and P. Fischer, Sci. Adv., 2015, 1, 1500501.

35 P. Zijlstra, P. M. R. Paulo and M. Orrit, Nat. Nanotechnol., 2012, 7, 379-382.

36 W. Ma, H. Kuang, L. Xu, L. Ding, C. Xu, L. Wang and N. A. Kotov, Nat. Commun., 2013, 4, 3689.

37 Y. He, K. Lawrence, W. Ingram and Y. Zhao, Chem. Commun., 2016, 52, 2047-2050.

38 E. Hendry, T. Carpy, J. Johnston, M. Popland, R. V. Mikhaylovskiy, A. J. Lapthorn, S. M. Kelly, L. D. Barron, N. Gadegaard and M. Kadodwala, Nat. Nanotechnol., 2010, 5, 783-787.

39 K. M. McPeak, C. D. van Engers, S. Bianchi, A. Rossinelli, L. V. Poulikakos, L. Bernard, S. Herrmann, D. K. Kim, S. Burger, M. Blome, S. V. Jayanti and D. J. Norris, Adv. Mater., 2015, 27, 6244-6250.

40 Y. Choi, T. Kang and L. P. Lee, Nano Lett., 2009, 9, 85-90.

41 J. Li, S. K. Cushing, F. Meng, T. R. Senty, A. D. Bristow and N. Wu, Nat. Photonics, 2015, 9, 601-607.

42 M. L. Brongersma, N. J. Halas and P. Nordlander, Nat. Nanotechnol., $2015,10,25-34$. 